\title{
Renormalization and black hole entropy in Loop Quantum Gravity
}

\author{
Ted Jacobson 1 \\ Department of Physics \\ University of Maryland \\ College Park, MD 20742-4111, USA
}

\begin{abstract}
Microscopic state counting for a black hole in Loop Quantum Gravity yields a result proportional to horizon area, and inversely proportional to Newton's constant and the Immirzi parameter. It is argued here that before this result can be compared to the BekensteinHawking entropy of a macroscopic black hole, the scale dependence of both Newton's constant and the area must be accounted for. The two entropies could then agree for any value of the Immirzi parameter, if a certain renormalization property holds.
\end{abstract}

The number of microscopic states of a black hole has been computed in Loop Quantum Gravity (LQG), in the state space of spin networks. The result for the entropy of a black hole with horizon area $A$ is

$$
S_{\mathrm{LQG}}=\frac{b}{\gamma} \frac{A}{\hbar G},
$$

where $b$ is a numerical constant and $\gamma$ is the Immirzi parameter. These calculations have a long and continuing history (see for example [1, 2, 3, 4, 5, [6, 17, 8, 9, 10] and for reviews [11, 12, 13]), including some controversy over the correct evaluation of the number of states. The results differ only in the value of $b$ however (unless states related by surface diffeomorphisms are identified, as has discussed for example in [14]). In addition to the case of spherically symmetric, static black holes, the result (1) has been shown to hold, with the same value of $b$, in the presence of scalar, Maxwell, and Yang-Mills fields [15]

\footnotetext{
${ }^{1}$ E-mail: jacobson@umd.edu
} 
as well as for spinning black holes in pure GR [16]. According to current understanding, the Immirzi parameter represents a quantization ambiguity in LQG, which affects physical observables. That is, it is generally believed that different values of $\gamma$ define physically different quantum theories.

Most discussions of the entropy observe that in order for $S_{\mathrm{LQG}}$ to match the Bekenstein-Hawking entropy $S_{\mathrm{BH}}=A / 4 \hbar G$, the Immirzi parameter must be chosen to have a special value,

$$
\gamma=4 b
$$

Not much is said about why $\gamma$ should have this particular value, but the idea seems to be that one can regard this calculation as a derivation of the "correct" value. Since any Immirzi parameter may be adopted in the microscopic theory, such an interpretation can make sense only if, as suggested for example in [11], this is the unique value for which the theory possess the classical limit of general relativity.

While it may be a logical possibility that the existence of a classical GR limit requires a unique value of $\gamma$, I can see no reason other than the entropy calculation to suspect that. Moreover, it is hard to see how the entropy computations carried out so far could probe LQG deeply enough to ascertain such a consistency condition for the continuum limit. The reason is that these computations involve only the kinematics, not the dynamics of the theory. Thus, it seems to me more reasonable to expect that, if the microscopic calculation of black hole entropy is really correct, then it should agree with $S_{\mathrm{BH}}$ for all values of $\gamma$.

On the face of it this looks impossible, however in fact the comparison of $S_{\mathrm{LQG}}$ with $A / 4 \hbar G$ has been made prematurely. The latter refers to a property of a semiclassical black hole. As such, the area is measured using the low energy effective metric field, and the Newton constant is the low energy effective Newton constant. By contrast, the area and Newton constant in $S_{\mathrm{LQG}}$ are the microscopic quantities appearing in the fundamental formulation of the theory [17]. This raises the question of exactly how the "correspondence principle" between the microscopic description in LQG and the effective field theory (EFT) description operates.

Anything said about the correspondence now is necessarily provisional, since fundamental aspects of LQG have not yet been understood. The Hamiltonian constraint, which encodes all the dynamics, remains to be understood, much less solved. Once solved, the theory can in principle only make statements about diffeomorphism invariant observables, and only tentative first 
steps have been achieved for the identification of a suitable class of such observables. Nevertheless, the setting of the black hole entropy computations is well-defined, and reasonably well-motivated, at least enough to merit scrutiny of its theoretical basis and self-consistency.

We now come to the central point of this note: there is no reason to expect the correspondence between microscopic and macroscopic quantities to be trivial. There are two different reasons for this. One is that in translating from a discrete spin-network to a field theoretic framework there is a profound change of objects and language. The other, independent reason is that even once the correspondence to an effective field theory has been made, the renormalization group flow of that theory from the UV to the IR is highly nontrivial. What then can be said?

Let us initially consider pure gravity, for simplicity, and let us assume that the microscopic cosmological constant $\Lambda_{\text {micro }}$ is zero and that an EFT limit exists. Dimensional analysis alone then implies that the low energy effective couplings are related to the microscopic parameters via

$$
\Lambda_{\mathrm{eff}}=f(\gamma) / \hbar G_{\mathrm{micro}}, \quad G_{\mathrm{eff}}=g(\gamma) G_{\mathrm{micro}},
$$

where $f(\gamma)$ and $g(\gamma)$ are at this stage unknown functions of the Immirzi parameter.

The relation between the areas assigned to a given surface, at the macro and micro levels of description, is only constrained by dimensional analysis to have the form

$$
A_{\text {eff }}=k\left(\gamma, A_{\text {micro }} / \hbar G_{\text {micro }}\right) A_{\text {micro }},
$$

where $k$ is an unknown function of its dimensionless arguments. However, since the area should be additive with respect to a decomposition of the surface into parts, it seems that $A_{\text {eff }}$ must scale linearly with $A_{\text {micro }}$. Moreover, if the areas are not proportional, then the microscopic entropy does not scale with the macroscopic area, so cannot possibly agree with $S_{\mathrm{BH}}$. Since I merely want to ask if it is theoretically possible that the entropies do agree for all values of $\gamma$, I will therefore assume the areas are related as

$$
A_{\text {eff }}=h(\gamma) A_{\text {micro }}
$$

The notion of area renormalization may at first appear alien, but note that the macro and micro "structure" of a surface are in principle quite different notions. (For an approach to the notions of coarse-graining and area 
renormalization in LQG see [18].) Moreover, on the EFT level, one expects a nontrivial relation between the low energy effective metric field and the metric at a UV cutoff scale (see e.g. [19] and references therein).

Although $f, g$ and $h$ relate quantities in the discrete and continuous frameworks, they are similar to the usual renormalization constants of QFT, so I will call them by that same name. The relation for the effective cosmological constant $\Lambda_{\text {eff }}$ has been indicated in (3), but I will say no more about it here except to assume that $\hbar G_{\text {eff }} \Lambda_{\text {eff }} \ll 1$, i.e. that the cosmological constant is very small in units of the effective Planck length, so that it may be ignored from here on.

In writing these relations, and in the rest of this paper, I am simply assuming that a semiclassical EFT limit of LQG exists, for at least some $\gamma$. The problem of understanding these renormalization relations is a crucial part of understanding the semiclassical limit of LQG. While this is a wide open problem, it can nevertheless be asked at present what properties must they exhibit if the existing microscopic black hole entropy calculations are to be valid?

The Bekenstein-Hawking entropy $S_{\mathrm{BH}}$ of a macroscopic black hole is expressed in terms of the "renormalized" quantities as

$$
S_{\mathrm{BH}}=\frac{A_{\mathrm{eff}}}{4 \hbar G_{\mathrm{eff}}}
$$

while the microscopic LQG result (1) should be written as

$$
S_{\mathrm{LQG}}=\frac{b}{\gamma} \frac{A_{\text {micro }}}{\hbar G_{\text {micro }}} .
$$

These will agree provided

$$
\gamma=4 b \frac{A_{\text {micro }} / G_{\text {micro }}}{A_{\text {eff }} / G_{\text {eff }}}
$$

The relation between the effective and microscopic quantities must therefore be taken into account.

In terms of the renormalization constants defined in (3) and (5), condition (8) becomes

$$
\gamma=4 b \frac{g(\gamma)}{h(\gamma)}
$$


Condition (9) may have (i) no solutions, (ii) a discrete set of solutions, or (iii) a continuous range of $\gamma$ might be solutions. Without knowing more about $g(\gamma) / h(\gamma)$, we can not say which of these is the case. If there are no solutions, then $S_{\mathrm{BH}}$ is never recovered, from which we infer that $S_{\mathrm{LQG}}$ can not in fact be the correct black hole entropy in LQG. If there is instead a discrete set of solutions, we are back in the position of having to maintain that, for some mysterious reason, the counting works only for special values of $\gamma$. The final possibility is that a continuous range of values of $\gamma$ are solutions to (9).

If a range of values of $\gamma$ solve (9), then there must be a tight relation between the renormalization of area and Newton's constant. That is, the ratio $g(\gamma) / h(\gamma)$ must be proportional to $\gamma$, and with the particular coefficient $1 / 4 b$. Is this conceivable?

If the area is not renormalized, i.e. if $h(\gamma) \equiv 1$, then (9) would imply $g(\gamma)=\gamma / 4 b$. It is not easy to imagine that the renormalization of Newton's constant is simply proportional to $\gamma$, nor that it always has this form, with the same coefficient, independent of the matter (Maxwell field, etc.) that is coupled to the metric. In fact, from the EFT side it is clear that coupling to different sorts of matter must alter the renormalization of $G$. Therefore it appears untenable that, without area renormalization, the LQG entropy computation yields $S_{\mathrm{BH}}$ for all values of $\gamma$ (and for generic matter).

Area renormalization significantly changes the picture however. The renormalization of $G$ can then be arbitrarily complicated, and dependent on the matter content, as long as it is universally tied to the renormalization of the area operator in the appropriate fashion indicated by (9). I see no reason why this could not be the case. If the continuum limit indeed exists, and the LQG black hole state counting is correct, then I would assert that it must be the case.

I have indicated a scenario in which $S_{\mathrm{LQG}}$ correctly counts the black hole entropy, without the need to select a special Immirzi parameter. It involves an unproven hypothesis about the correspondence between LQG and the semiclassical EFT of GR. Can this hypothesis be tested?

One thing that can be done is to ask whether it could possibly be robust allowing for additional theory parameters. For instance, if a microscopic cosmological constant $\Lambda_{\text {micro }}$ is included, then a dimensionless quantity $\lambda=\hbar G_{\text {micro }} \Lambda_{\text {micro }}$ exists, and the renormalization constants can depend upon $\lambda$. Another example of a parameter would be a gauge coupling constant. Allowing for some collection of such parameters would change nothing essential in the above discussion, so the required robustness seems at least possible. 
However, this does make clear that if the hypothesis is valid, it must be due to a very general feature of the emergence of the EFT description.

To directly test the hypothesis requires an improved understanding of the EFT limit, but need not necessarily involve black holes. Although the numerical constant $b$ is the one that arises in the horizon state counting, it does so in a way that might be more generally relevant to the relation between semiclassical geometry of areas and the microscopic, spin network

variables. Thus perhaps the validity of the relation (9) can be tested in a simpler setting, for example even in the vacuum.

\section{Acknowledgments}

I am grateful to Kirill Krasnov, Don Marolf, Lee Smolin, and Aron Wall for useful comments and discussions on a draft of this paper. This work was supported in part by the National Science Foundation under grant PHY0601800 .

\section{References}

[1] K. V. Krasnov, "Counting surface states in the loop quantum gravity," Phys. Rev. D 55, 3505 (1997) arXiv:gr-qc/9603025].

[2] C. Rovelli, "Black hole entropy from loop quantum gravity," Phys. Rev. Lett. 77, 3288 (1996) arXiv:gr-qc/9603063.

[3] A. Ashtekar, J. Baez, A. Corichi and K. Krasnov, "Quantum geometry and black hole entropy," Phys. Rev. Lett. 80, 904 (1998) arXiv:gr-qc/9710007.

[4] M. Domagala and J. Lewandowski, "Black hole entropy from quantum geometry," Class. Quant. Grav. 21, 5233 (2004) arXiv:gr-qc/0407051.

[5] K. A. Meissner, "Black hole entropy in loop quantum gravity," Class. Quant. Grav. 21, 5245 (2004) arXiv:gr-qc/0407052.

[6] O. Dreyer, F. Markopoulou and L. Smolin, "Symmetry and entropy of black hole horizons," Nucl. Phys. B 744, 1 (2006) arXiv:hep-th/0409056]. 
[7] A. Ghosh and P. Mitra, "An improved lower bound on black hole entropy in the quantum geometry approach," Phys. Lett. B 616, 114 (2005) arXiv:gr-qc/0411035.

[8] T. Tamaki and H. Nomura, "Ambiguity of black hole entropy in loop quantum gravity," Phys. Rev. D 72, 107501 (2005) arXiv:hep-th/0508142].

[9] A. Corichi, J. Diaz-Polo and E. Fernandez-Borja, "Quantum geometry and microscopic black hole entropy," Class. Quant. Grav. 24, 243 (2007) arXiv:gr-qc/0605014.

[10] T. Tamaki, "Considering boundary conditions for black hole entropy in loop quantum gravity," Class. Quant. Grav. 24, 3837 (2007) arXiv:0707.0341 [hep-th]].

[11] A. Ashtekar and J. Lewandowski, "Background independent quantum gravity: A status report," Class. Quant. Grav. 21, R53 (2004) arXiv:gr-qc/0404018.

[12] T. Thiemann, "Loop quantum gravity: An inside view," arXiv:hep-th/0608210.

[13] A. Corichi, J. Diaz-Polo and E. Fernandez-Borja, "Loop quantum gravity and Planck-size black hole entropy," J. Phys. Conf. Ser. 68, 012031 (2007) arXiv:gr-qc/0703116.

[14] A. Alekseev, A. P. Polychronakos and M. Smedback, "On area and entropy of a black hole," Phys. Lett. B 574, 296 (2003) arXiv:hep-th/0004036.

[15] A. Ashtekar, J. C. Baez and K. Krasnov, "Quantum geometry of isolated horizons and black hole entropy," Adv. Theor. Math. Phys. 4, 1 (2000) arXiv:gr-qc/0005126.

[16] A. Ashtekar, J. Engle and C. Van Den Broeck, "Quantum horizons and black hole entropy: Inclusion of distortion and Class. Quant. Grav. 22, L27 (2005) arXiv:gr-qc/0412003.

[17] I have been discussing the relevance of renormalization of $G$ for black hole entropy computations in LQG for over a decade in talks and privately 
(my early remarks were mentioned in Ref. [2]). An important new element of the present paper is the allowance for area renormalization, a possibility suggested to me (by someone I do not currently recall) at an ITP program in 1999.

[18] E. R. Livine and D. R. Terno, "Quantum black holes: Entropy and entanglement on the horizon," Nucl. Phys. B 741, 131 (2006) |arXiv:gr-qc/0508085]; E. R. Livine and D. R. Terno, "Reconstructing quantum geometry from quantum information: Area renormalisation, coarse-graining and entanglement on spin networks," arXiv:gr-qc/0603008.

[19] M. Reuter and J. M. Schwindt, "Scale-dependent metric and causal structures in quantum Einstein gravity," JHEP 0701, 049 (2007) arXiv:hep-th/0611294. 